\title{
The Fourier Transform Used as a Tool to Evaluate Systems for Digitizing Electron Images
}

\author{
Patrick Bron, Jean Paul Rolland and Daniel Thomas \\ Biologie Cellulaire et Reproduction, URA CNRS No 256, Université de Rennes 1, \\ Campus de Beaulieu, 35042 Rennes Cedex, France
}

(Received September 13; accepted September 30, 1996)

PACS.07.07.Hj - Display and recording equipment, oscilloscopes, TV cameras, etc.
PACS.87.64.Dz - Electron microscopy; scanning tunneling and atomic force microscopy

\begin{abstract}
Image processing methods are commonly used for the structure determination of biological molecules using electron microscopy. However, the first and crucial step is to convert the electron image intensities into numerical values to be further processed with the computer. We propose a simple method based on the Fourier transform to evaluate characteristics of different digital acquisition systems frequently found in laboratories, from the video camera linked to the electron microscope to desktop publishing scanners. Such systems have been chosen in order to represent a wide range of devices and prices.
\end{abstract}

\section{Introduction}

As a result of advances in the field of computer science and with the availability of low cost powerful computers, electron image analysis can be performed without much difficulties, even using highly sophisticated algorithms. Therefore, the extracted information from a single image could vary in a quantitative and qualitative manner [1,2], regarding to the shape, contrast or texture of the studied specimen. Electron microscopy has a wide application field, from a high resolution structural study of crystals in material science to a low resolution evaluation of immunogold labelling in biology. However the crucial step is to convert the optical density distribution across the image to a numerical matrix representation. This operation needs the use of a digitizing device which has to fulfill several criteria: efficiency of information transfer in terms of detection, dynamic and resolution, and also capacity to digitize large size images.

Presently, the electron micrograph is still the best system for image recording, considering its high sensitivity to the electron signal for an affordable price. Consequently, most of this work is focused on systems which use the electron micrograph as the original image support.

Many possibilities for digitizing an image are now available, they can be classified as follow: spot or slide scanning captors, and bidimensional captors. Following this classification, we have evaluated the characteristics of a wide range of acquisition devices frequently found in laboratories, from the scanning microdensitometer to desktop publishing scanners. For comparison, we used a video camera linked to the electron microscope permitting to record 
an electron image into a video image, subsequently converted into a digital format. For this work, images of catalase crystals were recorded, one negative was selected, digitized and its Fourier transform was computed.

\section{Materials and Methods}

Image of negative stained Catalase crystals from Agar have been recorded on Kodak So (163) film using a CM12 Philips Electron Microscope operated at $80 \mathrm{kV}$ and with a nominal magnification of 35000 . Micrographs were evaluated for focus, astigmatism, and drift by optical diffraction. To standardize measurements, a picture presenting a limited astigmatism and a first minimum of the transfer function between $1 / 8$ and $1 / 7 \AA$ was selected and used for digitizing.

\subsection{Digitizing}

Digitizing was performed using the following apparatus.

\subsubsection{Scanning Microdensitometer}

The electron micrograph was digitized with an Optronic rotating drum densitometer with a raster step of $12.5 \mu \mathrm{m}$ corresponding to $0.35 \mathrm{~nm}$ on the specimen scale.

\subsubsection{Leafscan 45}

Leafscan 45 is a scanner used mostly in art graphic and photography, composed by a CCD slide. The sampling was $0.4 \mathrm{~nm}$ on the specimen scale.

\subsubsection{CCD Cameras}

We used two CCD cameras: an Eikonix and a CCD digital Kodak Megaplus 1.4 I. The sampling was $0.4 \mathrm{~nm}$ on the specimen scale.

\subsubsection{Desktop Scanner in Transmission Mode}

An Agfa Arcus 2 scanner was used in transmission acquisition mode with a sampling size of 1800 d.p.i. which corresponds to a pixel size of $0.4 \mathrm{~nm}$ on the specimen.

\subsubsection{Desktop Scanner in Reflection Mode}

A paper print from the negative with a photographic enlargement of $\times 2.5$ was used for scanning. Two desktop scanners were assessed: an Agfa Arcus, and a UMAX UC 1200 SE ultravision. In both cases, the sampling size was 700 d.p.i., thus equivalent to $0.4 \mathrm{~nm}$ on the specimen scale.

Finally these images were compared to an image recorded from the electron microscope using a video camera CF1500 Elca (Sofretec) linked to a microcomputer fitted with a digital acquisition card Piter 500. The video camera has a circular target of $15 \mathrm{~mm}$ diameter, the digital acquisition card has an array size of 512 by 512 pixels, thus for a nominal magnification of 35000 on the microscope, the pixel size was of $0.8 \mathrm{~nm}$.

\subsection{Image Analysis}

Evaluation of the images characteristics was performed on the reciprocal space by computing the image Fourier transform. This operation was carried out on Sun workstations using dedicated softwares: SPIDER [3], IBIS [4], and MRC image processing programs [5]. 

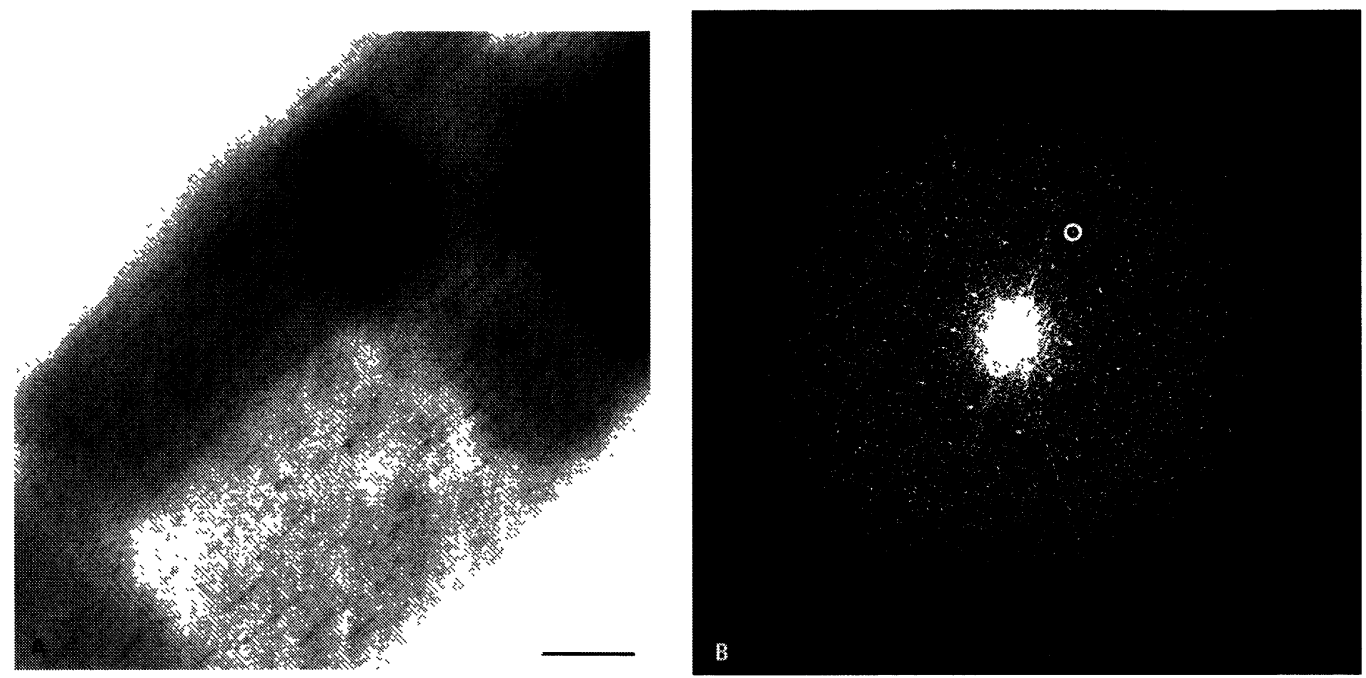

Fig. 1. - a) Electron image of a negatively stained crystal of catalase. Scale bar $=0.2 \mu \mathrm{m}$. b) Optical diffraction pattern from the catalase electron image. The $(0,6)$ reflection located at $1 / 14.6 \AA^{-1}$ has been encircled.

The Fourier transform was calculated on a $512 \times 512$ pixels catalase image area, the corresponding power spectrum was computed and displayed. From the power spectrum, a radial profile of the intensities was calculated by effecting a rotational averaging of the intensities along the radius; a Gaussian filter was then applied on the resulting profile. The relative Modulation Transfer Function (rMTF) was computed as the ratio of the amplitude of a given diffraction spot to the amplitude of a diffraction spot used as reference. Spot indexed $(2,0)$ was chosen as reference.

For comparison, a radial density profile was obtained from the optical diffraction negative, by scanning a line along the diameter of the diffraction ring using an analogue Joyce Loeble densitometer (MK III C S).

\section{Results}

The optical diffraction image carried out on the catalase electron micrograph (Fig. 1a) is shown in Figure $1 \mathrm{~b}$. The first minimum is located between $1 / 8$ and $1 / 7 \AA$. Diffraction spots can be seen towards the sixth order. The density profile corresponding to the optical spectrum is presented in Figure 4a.

\subsection{Scanning Microdensitometer}

The computed diffraction pattern (Fig. 2a) is very similar to the optical diffraction image. The slight astigmatism is retrieved, discrete spots are clearly resolved and extend to the sixth order. After a plateau, the intensity of the power spectrum falls gradually toward the first 


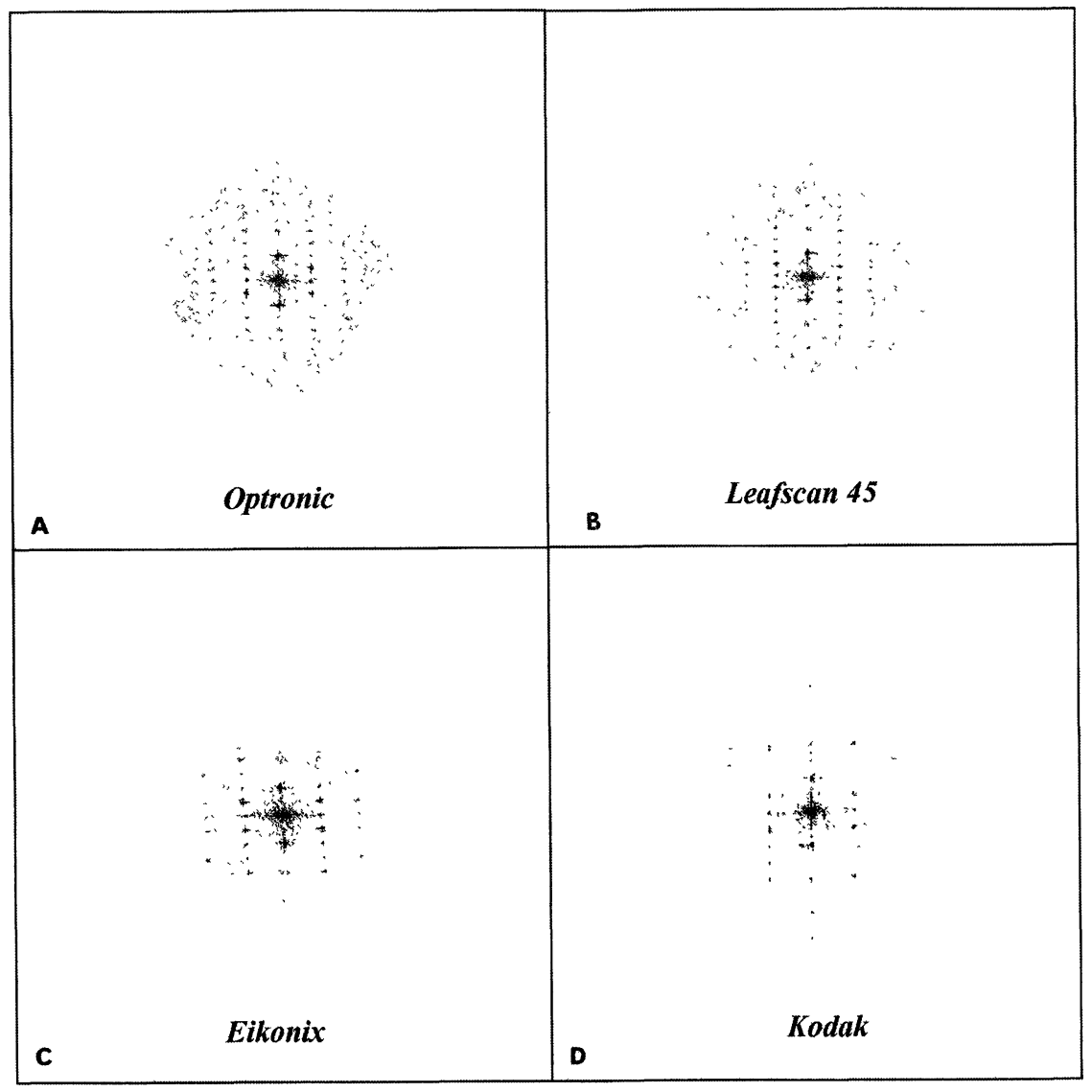

Fig. 2. - Power spectrums computed from the catalase electron micrograph digitized with the following apparatus: a) Optronic microdensitometer; b) Leafscan 45; c) CCD Eikonix camera; d) CCD Kodak camera. Scale (a): $0.65 \mathrm{~cm}=0.055 \AA^{-1} ;(\mathrm{b}): 0.65 \mathrm{~cm}=0.058 \AA^{-1} ;(\mathrm{c}): 0.65 \mathrm{~cm}=0.046 \AA^{-1}$; (d): $0.65 \mathrm{~cm}=0.043 \AA^{-1}$.

minimum of the transfer function (Fig. 4b). The rMTF is high and still significant in the high frequencies (Fig. 5a).

\subsection{Leafscan 45 Scanner}

The Leafscan scanner image provides a diffraction pattern where spots are clearly resolved above the background and where the first minimum ring is clearly observable (Fig. $2 \mathrm{~b}$ ). The power spectrum intensity is characterized by a plateau which falls down around $1 / 8 \AA$ as far as the first minimum (Fig. 4b). The rMTF is lower than the Optronic one for the low frequencies, but reaches a comparable level for high frequencies (Fig. 5a).

\subsection{CCD Cameras}

Eikonix and Kodak CCD camera present a diffuse scattered spectrum of regular shape in both space direction (Figs. 2c, d). Diffraction spots up to the 4th order in K and up to the 6th order 


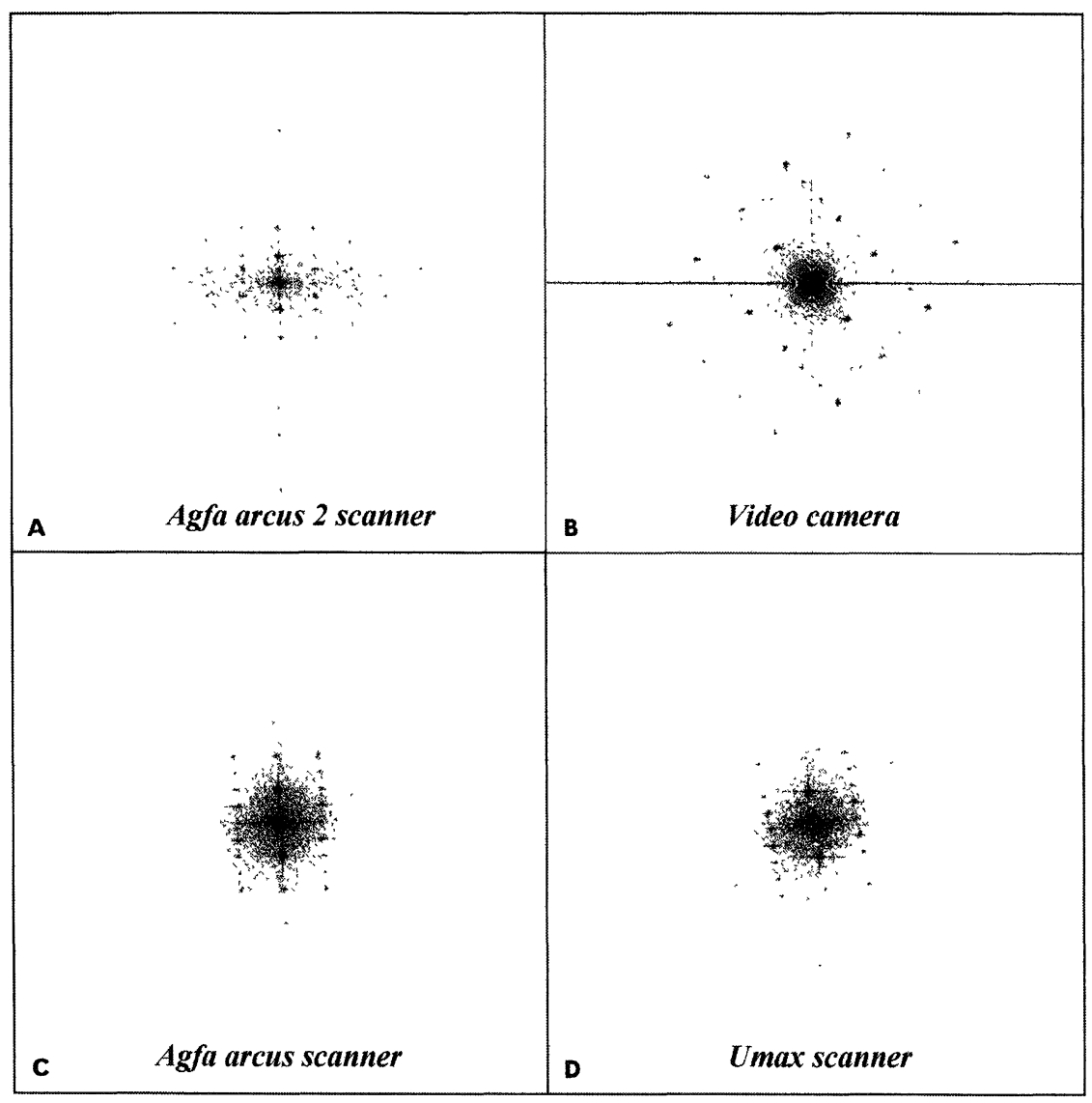

Fig. 3. - Power spectrums computed from electron micrograph digitized with the following apparatus: a) Agfa arcus 2 scanner in transmission operating mode; b) video camera linked to the microscope; c) Agfa arcus and (d) Umax scanner in reflection mode, Scale (a): $0.65 \mathrm{~cm}=0.05 \AA^{-1}$; (b): $0.65 \mathrm{~cm}=0.027 \AA^{-1} ;(\mathrm{c}, \mathrm{d}): 0.65 \mathrm{~cm}=0.032 \AA^{-1}$.

in $\mathrm{H}$ are clearly seen. The power spectrum intensity profile of the Eikonix camera shows a plateau between $1 / 16 \AA$ and $1 / 8 \AA$ followed by a marked intensity decrease to $1 / 6.5 \AA$. Instead of a plateau, the Kodak camera presents a mostly progressive decrease of intensity until $1 / 6.5 \AA$. In both cases, no ring corresponding to the first minimum can be seen. The Kodak camera presents a rMTF profile similar in shape to the Optronic one, but with significant lower values, while the Eikonix rMTF presents a dramatic decrease which rapidly reaches low values for frequencies higher than $1 / 40 \AA$ (Fig. 5a).

\subsection{Desktop Scanner in Transmission Mode}

When using an AGFA Arcus scanner, an important anisotropy is observed in the diffraction pattern, besides a repetition of the central pattern can be seen in both directions (Fig. 3a). The rMTF falls down rapidly to low values for frequencies higher than $1 / 40 \AA$ (Fig. 5b). 

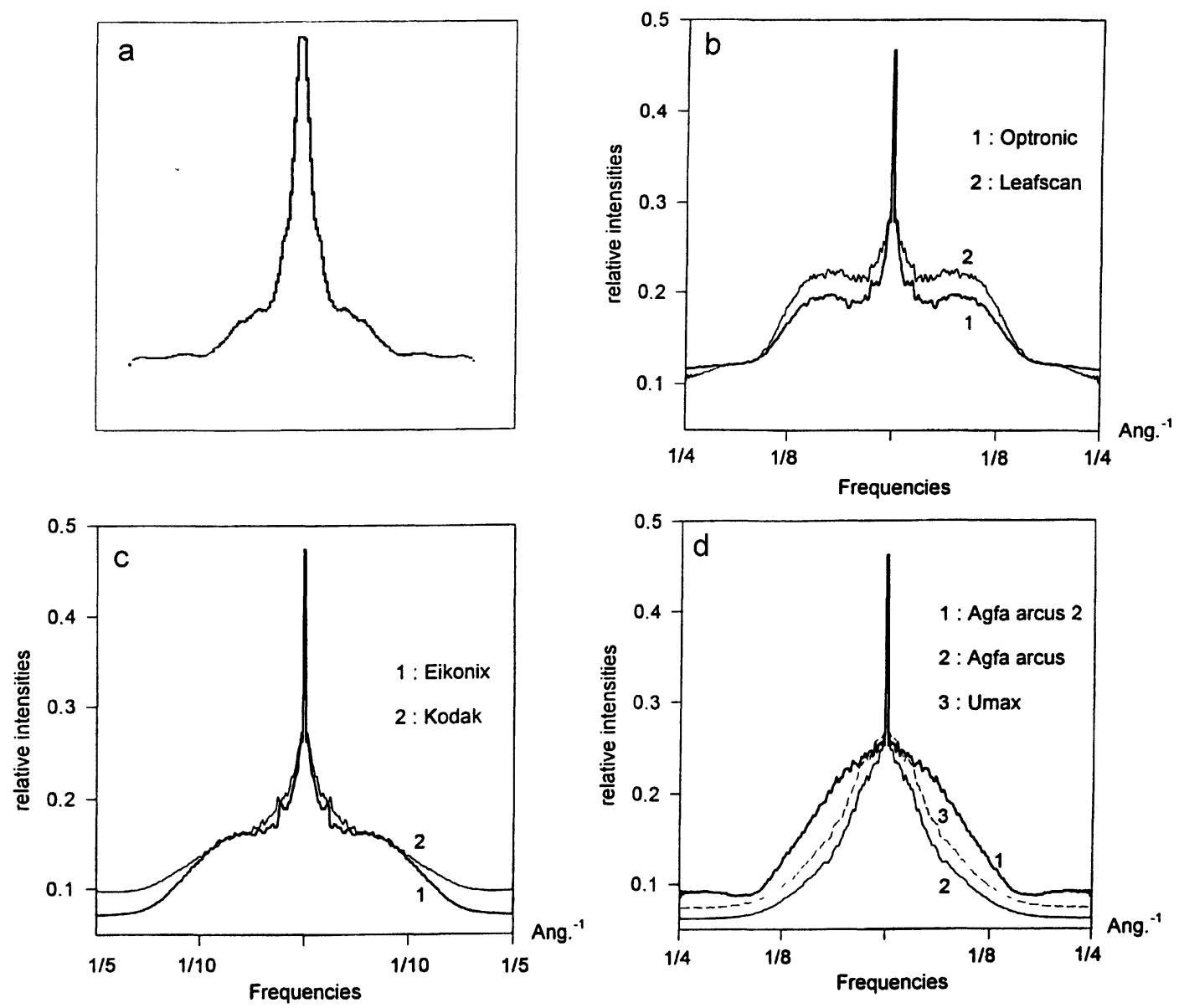

Fig. 4. - Radial profiles of the power spectrum: a) Optical diffraction; b) Optronic and Leafscan 45; c) Eikonix and Kodak CCD camera; d) Agfa arcus 2, Agfa arcus, Umax desktop scanners.

\subsection{Desktop Scanners in Reflection Mode}

The Agfa scanner provides a regular diffraction pattern apparently devoid of anisotropy. Diffraction spots up to the 6 th order in $\mathrm{H}$ and the 4 th order in $\mathrm{K}$ are seen, however an important background noise blurs the diffraction spots even at low frequencies (Fig. 3c).

The image from the UMAX scanner exhibits anisotropy clearly observed on the diffraction pattern. The repetition of the first order spots leads to the formation of discrete spots toward high frequencies (Fig. 3d).

For both scanner the spectrum intensity profile falls down very quickly (Fig. 4d). This is reflected by a low rMTF for frequencies higher than $1 / 60 \AA$ (Fig. 5b).

\subsection{Direct Digital Image Acquisition by a Video Camera}

When working at the same magnification as previously used, the video camera has a sampling twice coarser than the other systems tested, moreover the matrix size is limited to $512 \times 512$ pixels. The computed spectrum appears very noisy, but the diffraction spots are clearly well 

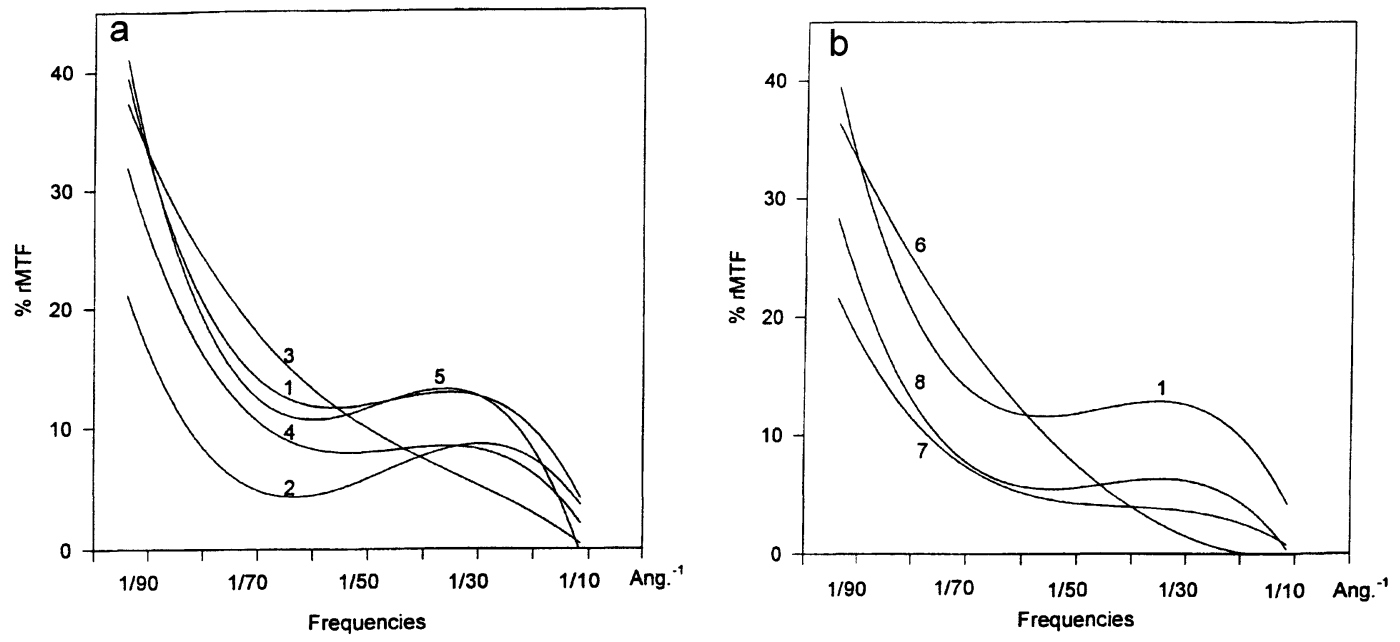

Fig. 5. - Relative modulation transfer function. a) 1: Optronic; 2: Leafscan 45; 3: CCD camera Eikonix; 4: CCD camera Kodak; 5: Video camera. b) 1: Optronic; 6: Agfa arcus 2; 7: Agfa arcus; 8: Umax scanner.

defined and stand out from background noise (Fig. 3b). This featuring is confirmed by a very high $\mathrm{rMTF}$, even for high frequencies (Fig. 5a). Due to the limited sampling size, the resolution is not better than $16 \AA$ and thus does not permit the observation of the first minimum ring.

\section{Discussion}

The quality of digital acquisition of an electron image is a key step in image processing. In material sciences, observations for high resolution studies are mostly performed at high magnification, while in biology, high resolution studies are limited in magnification of the range 35000-60000 as a result of the high sensitivity to the electron dose of biological specimens. Consequently, the requirements for digitizing images can differ between these two fields of investigation. For example, in biology, to preserve the resolution associated with the raw image, the sampling size will have to be adjustable as fine as necessary, but without inducing image alterations.

The lattice spacing of crystalline catalase is often used in biology, as an internal standard for calibration in electron microscopy [6]. Since catalase is organized in repeated motives of unit cells, we have used its intrinsic properties to estimate the quality of the image by a Fourier transform analysis: the discrete spectrum points allow to check rapidly the inner quality of the image, the first minimum ring of the transfer function represents the limit of the finest information directly accessible within the raw image, and the radial spectrum profile brings information related to the signal to noise ratio. Accordingly the resolution associated to the electron micrograph was estimated by optical diffraction between $1 / 8$ and $1 / 7 \AA$.

On digitized pictures, the first minimum of the transfer function was observed only on images which were digitized with the Optronic densitometer and with the Leafscan 45 scanner. Both systems have excellent physical characteristics, with a good rMTF for high frequencies. However the Optronic densitometer shows the best characteristics in terms of dynamic, but this system has a relatively slow speed for scanning and a sampling size at limited to $12.5 \mu \mathrm{m}$. 
Actually, microdensitometers are extremely accurate digitizing devices but could present yet some acquisition errors such as "pseudo-white noise" distribution of error [7].

With CCD cameras, the first minimum ring can not be observed in the power spectrum, however a weak decrease in intensity within the range of the $1 / 8 \AA$ frequencies is observed. For both cameras, the rMTF is very low for frequencies higher than $1 / 15 \AA$, limiting the possibility to retrieve high resolution. This phenomenon could be the result of high frequencies noise. Some variations in the sampling size could also occur, but this can be avoided by an accurate calibration of the camera bench. Nevertheless, CCD cameras are fast and easy to use for digitizing micrographs, even if they have a limited matrix size. The largest matrix available has $1024 \times 1024$ pixels, and consequently to work on larger area, the user has to merge different images.

When high resolution is not needed (i.e. for morphological analysis), using a desktop scanner could be a fast and cheap way for digitizing, yet differences exist between desktop scanners. Some scanners can induce anisotropy within the picture, as result of mechanical imprecisions. One of the scanners used avoids such problem, and allows to get a digitized image of good quality, however high frequencies informations are definitely lost. No improvement was observed when the same kind of scanner was used for digitizing the negative in transmission mode.

When an electron image is recorded by a video camera fitted on the microscope, we reach a better resolution than those obtained with a desktop scanner. The main limitation of the video camera system used in this study is the small array size of $512 \times 512$ pixels available with the digital acquisition card, however cards with matrix size of $1024 \times 1024$ or larger are now available. The sensitivity to electrons of these video cameras is presently good which allows to work on sensitive specimens with low electron doses, and to compute on line Fourier transform.

In this work, slow scan CCD cameras and imaging plates were not tested. Slow scan CCD camera offers a new way for electron image recording. These image detectors have outstanding properties, including a dynamic range, with near-perfect linearity, and negligible geometric distortion $[8,9]$. The current generation of these cameras is approaching single electron detection and delivers a typical image size of $1024 \times 1024$ pixels. Such camera allows a direct acquisition of electron diffraction pattern $[8,10]$, but still remains very expensive.

Recently developed by Fuji, the FDL 5000 system yields a digital image from an erasable imaging plate. According to the manufacturer this system presents a high linearity and sensitivity. Resolution on the imaging plate is about $4 \mu \mathrm{m}$ but read out pixel is only $25 \mu \mathrm{m}$ which could be limiting for high resolution studies at reduced magnifications.

In this paper, we propose a simple method based on the Fourier transform, to evaluate the qualities of digitizing devices. At a first glance, the power spectrum permits a quick evaluation of the global quality of the image. The radial profile derived from the power spectrum gives the information related to the noise induced by the apparatus, while the rMTF reflects the loss of information within the reciprocal space. The conclusions drawn apply only to the digitizing devices tested during this study, and thus are not representative of these models in general.

\section{Acknowledgments}

We are extremely grateful to G. Mosser, J.M. Carazo, J.L. Puteau, R. Wade and P. François who made us available their digitizing device and for many fruitful discussions. 


\section{References}

[1] Misell D.L., Image analysis, enhancement and interpretation. Practical Methods in Electron Microscopy, vol. 7 (North-Holland publishing company, 1978).

[2] Baker T.S., Image processing of biological specimens, in: "A bibliography in Electron Microscopy in Biology", J.D. Griffith, Ed. (Wiley, New York, 1981).

[3] Frank J., Shimkin B. and Dowse H., Ultramicroscopy 6 (1981) 343-358.

[4] Flifla J.M., Garreau M., Rolland J.P., Coatrieux J.L. and Thomas D., Comput. Applic. Biosci. 8 (1992) 583-586.

[5] Crowther R.A., Henderson R. and Smith J.M., J. Struct. Biol. 116 (1996) 9-16.

[6] Wrigley N.G., J. Ultrastructure Res. 24 (1968) 454-464.

[7] McGee P.A., Trus B.L. and Steven A.C., Micron 13 (1982) 221-228.

[8] De Ruijter W.J., Micron 26 (1995) 247-275.

[9] Ishizuka K., Ultramicroscopy 52 (1992) 7-20.

[10] Brink J. and Chiu W., J. Struct. Biol. 113 (1994) 23-34. 